\title{
Local and general anesthesia and premature extraction of deciduous teeth
}

\author{
Radosveta Andreeva ${ }^{1}$, Milena Georgieva ${ }^{1}$, Danail \\ Lichev $^{2}$, Evgeni Dimitrov ${ }^{1}$, Ani Belcheva ${ }^{3}$.
}
1. Department of Pediatric Dentistry, Medical University of Varna;

2. Department of Anestheology and Intensive Care, UMHAT "St.Marina", Varna;
3. Department of Pediatric Dentistry, Medical University of Plovdiv;

\begin{abstract}
The premature extraction under general anesthesia differs significantly from the same manipulation made with local anesthesia. The aim of the study is to compare the number, the type and the position of the prematurely extracted deciduous teeth in children treated with local and under general anesthesia. Subjects of monitoring were 60 children. The clinical group consists of 30 children with teeth for premature extraction under general anesthesia and the control group consists of 30 with children for premature extraction under local anesthesia. The dental status was examined and registered by the DMFT. The number and type of these teeth were calculated and registered in special cards. Results: The children from the first group had a greater average number of prematurely extracted teeth per child 3,22 $\pm 0,28$ and for the children from the second group that number is 1,03 \pm 0,07 . The children from the first group have 23 premature extracted anterior teeth. For the first group of children - 55 second temporary molars were prematurely extracted, in the second group -25 teeth. A greater number of deciduous teeth in the lower jaw in the two studied groups of children were prematurely extracted - 49 teeth in the first group and 43 teeth for the second group. Conclusion: These results demonstrate the connection between the carious activity, premature tooth loss and the need of prevention of younger children, especially with ECC, where the upper incisors are strongly damaged at a very young age.
\end{abstract}


Keywords: general anesthesia, premature loss, primary teeth

\section{Introduction}

For the overwhelming majority of children, successful behavior management in the dental office is accomplished through communicative behavior management techniques (tell-show-do, acclimatization, desensitisation, voice control, distraction, role modeling, positive reinforcement) and nitrous oxide. In this cases local anesthesia (LA) could be used for premature tooth extraction. In children where communicative techniques are unsuccessful, more advanced behavior management techniques include: passive immobilization, moderate sedation, and general anesthesia (GA). A 1991 study of 933 patients revealed the scope of caries, followed by behavior management problems and the patient's mental or physical condition as the most common reasons for dental treatment under general anesthesia $(1,2,3)$. The most common reasons cited for the treatment under GA of children with special needs are: extensive treatment needs, followed by negative behavior, and underlying medical condition 2 . Thus, the reasons for a patient's referral to have dental work completed while under general anesthesia are the same for children with and without special health care needs. "(GA) may be indicated for the patient who lacks the ability to cooperate, whether this is because of the child's age, anxieties, level of psychological maturity or presence of a medical, physical/mental disability or developmental delays. The decision to use GA must take into consideration alternative behavior management modalities, dental needs of the patient, the effect on the quality of dental care, the patient's emotional status, and the patient's medical status 13 " $(1,4)$. "The goal of general anesthesia in the pediatric dental patient is to eliminate cognitive, sensory, and skeletal motor activity to facilitate the delivery of quality comprehensive diagnostic, restorative, and/or other dental services. The indications for general anesthesia, according to the American Academy of Pediatric Dentistry (AAPD) (5), include the following: 1) patients who are unable to cooperate due to a lack of psychological or emotional maturity and/or mental, physical, or medical disability, 2) patients for whom local anesthesia is ineffective because of acute infection, anatomic variations, or allergy, 3) the extremely uncooperative, fearful, anxious, or uncommunicative child or adolescent, 4) patients requiring significant surgical procedures, 5) patients for whom the use of deep sedation or general anesthesia may protect the developing psyche and/or reduce medical risks, and 6) patients requiring immediate, comprehensive oral/dental care" $(2,6,7)$.

\section{Aim}

The aim of the study is to compare the number, the type and the position of the prematurely extracted deciduous teeth in children treated with local and under general anesthesia.

\section{Material and Methods}

Subjects of monitoring during the research were 60 children. The clinical (first) group consists of 30 children with teeth for premature extraction under general anesthesia and the control (second) group consists of 30 children for premature extraction under local anesthesia. All studied children are healthy, without any systemic diseases. Some of them were treated under general anesthesia due to immature age and extensive pathology. For the older children such a treatment is required because of their definitely negative behavior or dentofobia. All of the examined children were treated in the medico-dental center of Medical University, Varna, after the parents of each child signed an informed consent form.. The dental 
status was examined and registered according to the standards of WHO by the DMF $(T+t)$. This index is one of the most often used ones in oral epidemiology to estimate the distribution of dental caries and the need of treatment among the population. The index is based on clinical observation of patients with a dental mirror, a dental probe, cotton pellets and suction. The DMF $(T+t)$ index represents the sum of the teeth with decay-(D), missing teeth-(M), and those with fillings-(F). $T$ is tooth and $T+t$ is used for mixed dentition. The capital latter $\mathrm{T}$ marks the permanent dentition and the small $\mathrm{t}$ marks the temporary dentition (8). Special attention to the M (missing teeth) was paid. The primary teeth that have been extracted one (9), two (10) or more years before their physiological exfoliation, we define as prematurely extracted primary teeth. It means that we used the age criteria for premature tooth loss. Their number and type were calculated. The number and the type of the missing teeth and the teeth that have to be prematurely extracted of the examined children from the two groups (clinical and control) were filled in special registered cards for dental status.

\section{Results and Discussion}

There is a statistically significant difference between the average number of prematurely extracted deciduous teeth in children treated with local and those under general anesthesia - $t=5,79, p<0,05$ (Fig. 1).

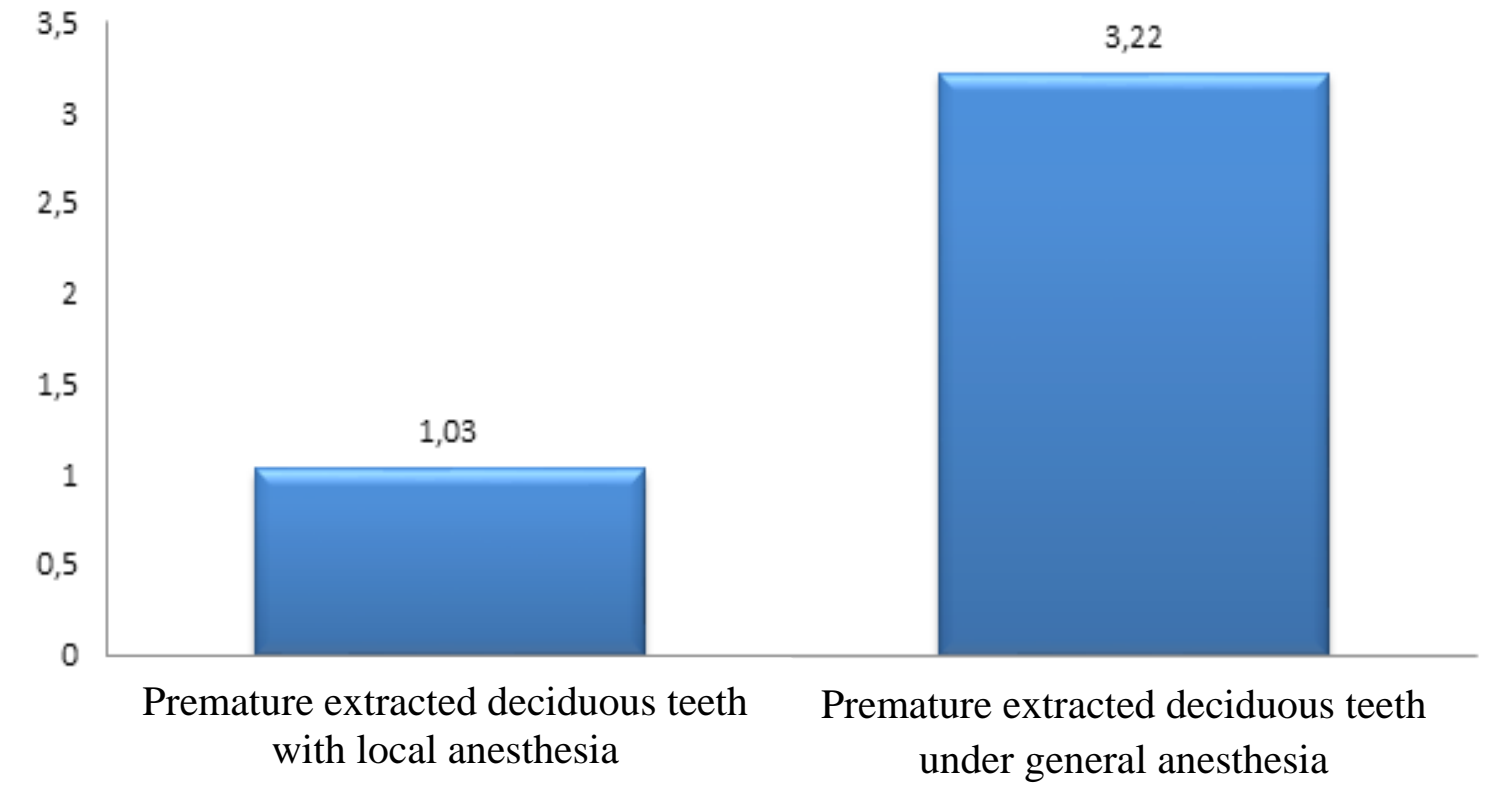

Fig. 1. Average number of prematurely extracted deciduous teeth per child treated with local anesthesia and general anesthesia

Children treated under general anesthesia had significantly greater average number of prematurely extracted teeth per child $3,22 \pm 0,28$, while in children treated with local anesthesia that number per child is $1,03 \pm 0,07$. The results indicate that children treated under general anesthesia have a significantly 
higher mean number of prematurely extracted primary teeth. This is explained by the fact that caries and their complications are the main cause of premature tooth loss, and these children have more carious lesions. Our results are similar to those reported by other authors $(11,12)$.

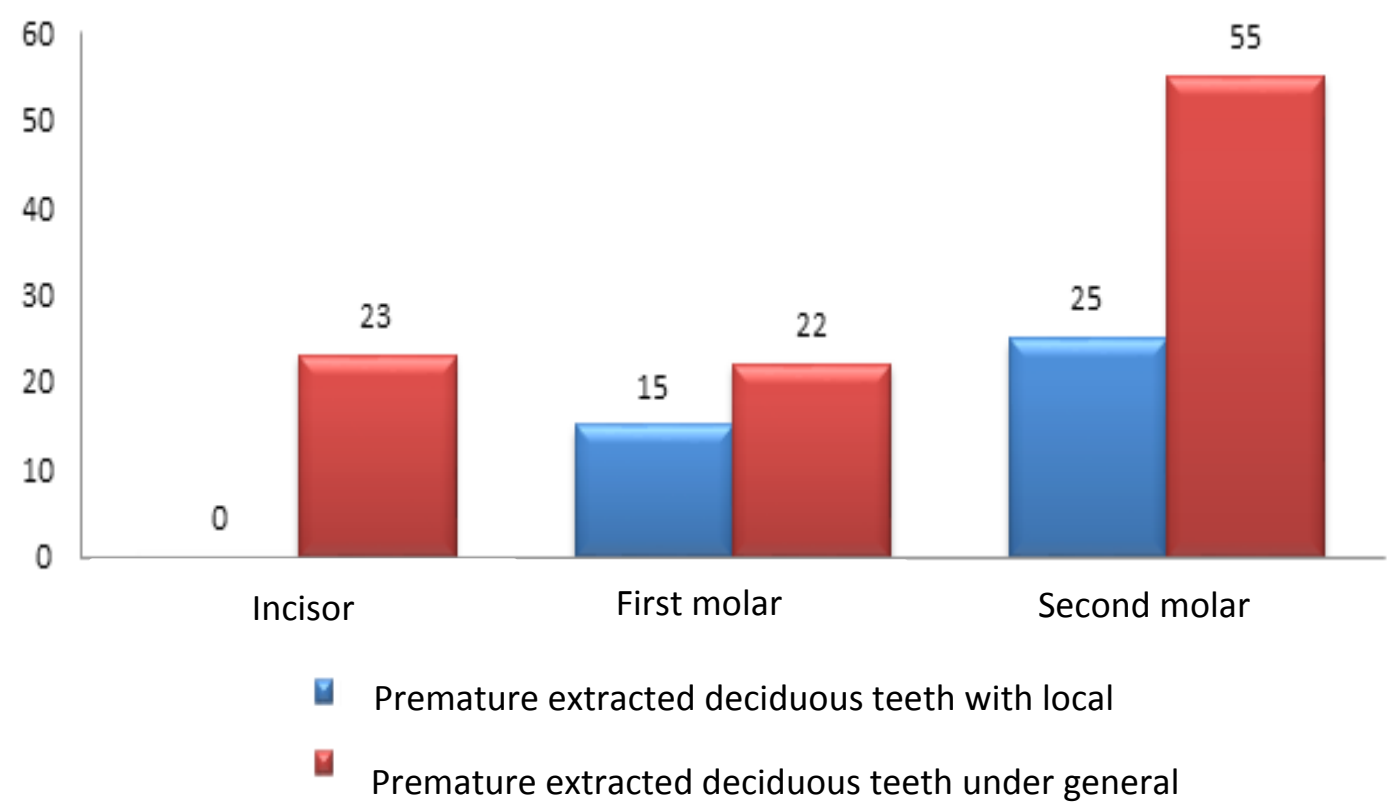

Fig. 2. demonstrates the difference in the type of prematurely extracted deciduous teeth between the two groups of studied children.

The results showed that children treated under general anesthesia have a significant number of prematurely extracted anterior teeth -23 teeth, while none of the children from the control group had prematurely extracted incisors. This could be explained by the fact that the children treated under general anesthesia are younger and most of them suffer from early childhood caries (ECC). This is a very aggressive, rapidly progressing form of caries that affects the vestibular surfaces of the temporary front teeth first. The second temporary molars constitute another group of teeth that have often been prematurely extracted. For the first group of children -55 second temporary molars were prematurely extracted, while in the second group the number of these teeth was 25. In both groups the second temporary molars were the most frequently prematurely extract deciduous teeth. The first temporary molars, which were prematurely extracted, were 22 in the first group, while in the second group, these teeth were 15 in number. For all types of teeth the differences between the two examined groups were statistically significant $-\mathrm{x} 2=12.8, \mathrm{p}<0.01$. Our results differ from those reported by other authors, who describe first molars as the most frequently prematurely extracted teeth under general anesthesia $(13,14)$.

Premature extractions of temporary teeth in the upper and lower jaws of children treated under local and general anesthesia are illustrated in Fig. 3. 


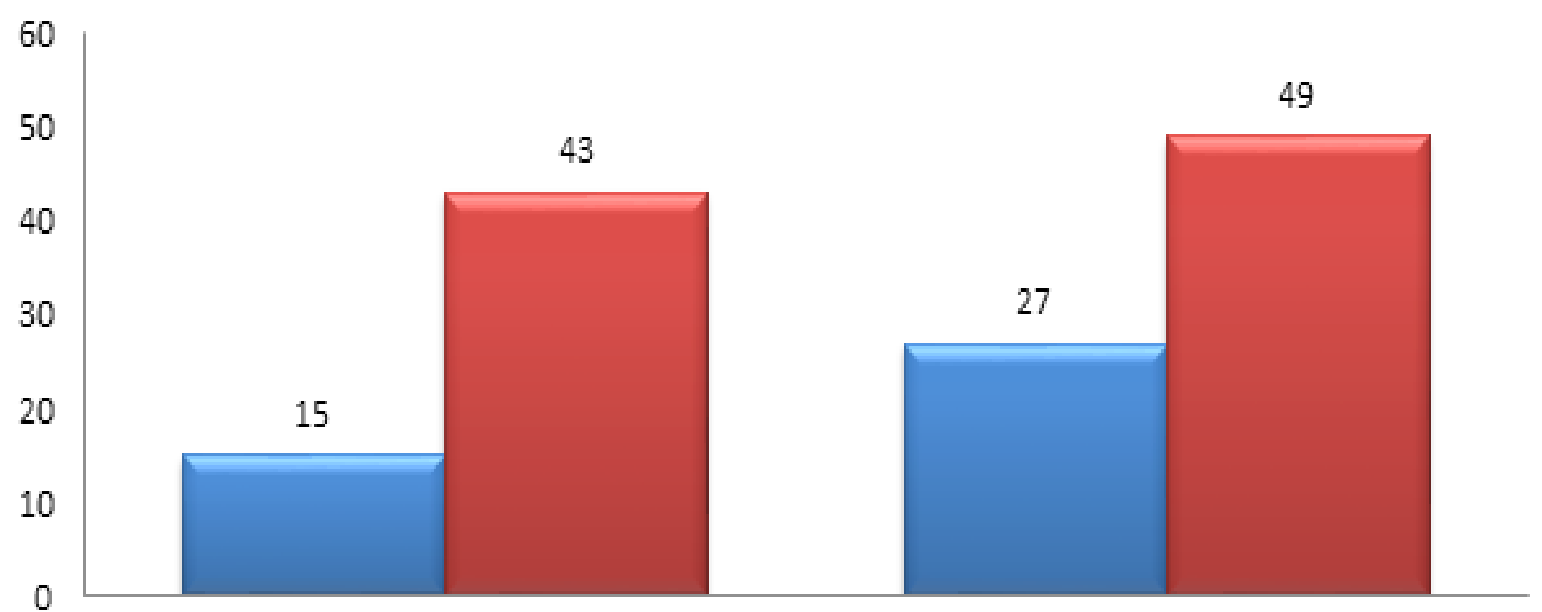

Upper jaw

Lower jaw

- Premature extracted deciduous teeth with local

- Premature extracted deciduous teeth under general

Fig. 3. Average number of premature extractions of temporary teeth in the upper and lower jaws of children treated under local and general anesthesia

The results of the research show a greater number of prematurely extracted deciduous teeth in the lower jaw in the two studied groups of children -49 teeth in the first group and 43 teeth for the second group. In the upper jaw that number is respectively 27 teeth for the first group and 15 for the second group. The differences in terms of the number of prematurely extracted deciduous teeth in each jaw for both treatment groups were statistically significant $-\mathrm{x} 2=1.43, \mathrm{p}<0.05$. More frequent premature extractions in the lower jaw are associated with tooth decays in the lower jaw. Similar results have been reported by other authors $(15,16)$.

Children are premedicated with Midazolam. Under a general mask, inhalation anesthesia with Sevoflurane is applied, and an intravenous way is provided. Rocuronium and Propofol are introduced. Airway is maintained by oro or nasotracheal intubation. Oral tamponade is obligatory. Maintenance of the anesthesia is done with Sevoflurane and Fentanil. For local anesthesia Articaine is used, if and when necessary. After the treatment and extubation, the children are admitted to a day care hospital for observation. Anti-drainage therapy is recommended.

Treatment under general anesthesia is long (three hours on average), because the children treated in this way are usually with a large number of problematic teeth. The complete treatment is done in one session because of the specificity of the contingent - significantly immature children with ECC or definitely negative bigger children, whose behavior couldn't be managed in routine ways. 


\section{Conclusion}

The data from the oral examination status and the special registered cards show that children with premature extraction of temporary teeth under general anesthesia have a significantly higher number of prematurely extracted teeth in connection with a higher caries activity and the upper incisors are more often prematurely extracted compared to the control group (children with early loss of teeth, treated under local anesthesia). Thes results demonstrate the connection between carious activity, premature tooth loss and the need of prevention of younger children, especially with ECC, where the upper incisors are strongly damaged at a very young age.

\section{References}

1. Sheller B, Williams B, et al. Reasons for repeat dental treatment under general anesthesia for the healthy child. Pediatr Dent. 2003;25(6):546-552.

2. American Academy of Pediatric Dentistry. Policy on third-party reimbursement of medical fees related to sedation/general anesthesia for delivery of oral health services. Pediatr Dent Reference Manual. 2009-2010;31(6):72-73.

3. Joaquin de Nova Garcia, Lopez M, Nuria E, et al. Criteria for selecting children with special needs for dental treatment under general anesthesia. Med Oral Patol Oral Cir Bucal. 2007;12(1):E496-503.

4. American Academy of Pediatric Dentistry. Guidelines on behavior management for the pediatric patient. Pediatr Dent. 2008;31(6):132-140.

5. American Academy of Pediatric Dentistry. Guideline on the elective use of minimal, moderate, deep sedation and general anesthesia for pediatric dental patients. Pediatric Dentistry Reference Manual. 2006:110-118.

6. Savanheimo N, Vehkalahti M, et al. Reasons for and parental satisfaction with children"s dental care under general anesthesia. International Journal of Pediatric Dentistry. 2005;15:448-454.

7. Park M, Sigal M. The role of hospital-based dentistry in providing treatment for persons with developmental delay. JCDA. 2008;74:353-357.

8. Birute J, Jorma I. Treatment under general anesthesia among children younger than 6 years in Lithuania. Medicina (Kaunas). 2013;49(9);403-408.

9. Atanasov K. Early loss of some primary teeth and the influence upon their permanent sucssesors.Stomatologia.1977;1:47-52.

10. Owen DG. The incidence and nature of space closure following the premature extraction of deciduous teeth: a literature survey. Am J Orthod. 1971;59:37-49 
11. Fuhrer, C., Weddell, J., Sanders, B., Jones, J., Dean, J., Tomlin, A. Effect on behavior of dental treatment rendered under conscious sedation and general anesthesia in pediatric patients. Pediatric Dentistry, 31 (7). 2009:492-497.

12. Foster, T., Perinpanayagam, H., Pfaffenbach, A., \& Certo, M. Recurrence of early childhood caries after comprehensive treatment with general anesthesia and follow-up. J Dent child (chic). 73(1). 2006:25-30.

13. Amin, $M$ \& Harrison, R. Change in parental oral health practices following a child's dental treatment under general anaesthesia. Eur Arch Paediatr Dent, , 2006, 7(2):116-120.

14. Stapleton, M, Sheller, B, Williams, B \& Mancl, L. Combining procedures under general anesthesia. Pediatr Dent 2007 29(5):397-402

15. Costa, L, Harrison, R, Aleksejuniene, J, Nouri, M \& Gartner, A. factors related to postoperative discomfort in young children following dental rehabilitation under general anesthesia. Pediatr Dent. 2011, 33(4):321-326.

16. Peerbhay F, Barrie RB: The burden of early childhood caries in the Western Cape Public Service in relation to dental general anaesthesia: implications for prevention. SADJ. 2012, 67: 1419

\section{Corresponding author:}

Address for correspondence:

Radosveta Andreeva

Faculty of Dental Medicine,

Medical University of Varna

55 Marin Drinov Str.

9002 Varna, Bulgaria

Tel.:0887202 495

e-mail: doctor_ra@abv.bg 